INVESTIGACIÓN

Recibido: 15/09/2017 --- Aceptado: 11/03/2019 --- Publicado: 15/06/2019

\title{
SEMIDESNUDO, GÉNERO Y OTROS FACTORES EN PUBLICIDAD TELEVISIVA. UN ACERCAMIENTO DESDE LA NEUROCIENCIA
}

\section{Half-naked, gender and other factors on television spots. A neuroscience approach}

(1D) (8) $\mathbb{R}^{6}$ Alejandro Tapia-Frade1: Universidad Loyola. Andalucía, España. ajtapia@uloyola.es

Andrés del Toro-Acosta. Universidad Loyola. Andalucía, España. atoro@uloyola.es

\section{RESUMEN}

Este trabajo muestra los resultados de un experimento de neurociencia realizado sobre un grupo de 20 personas de ambos sexos, a las que se midió su actividad electrodérmica de forma simultánea mientras visionaban spots de televisión. Se prestó especial atención al análisis de diferencias de género y sus patrones de activación atencional y emocional. Los resultados ponen de manifiesto que los spots que mostraban modelos semidesnudos o en ropa interior resultan significativamente superiores en atención en ambos sexos respecto de los que no presentan esta característica. También se pudieron concluir diferencias significativas en relación a la familiaridad de los espectadores con la marca en ambos sexos (las marcas conocidas son atendidas en mayor medida). Finalmente, pudo también destacarse que la activación atencional se produce en mayor medida al inicio y finalización del spot, aunque se aprecia más claramente en el caso de las mujeres. De igual modo, se puede sugerir un patrón común de activación atencional cuando se produce una ruptura relevante de registro sonoro.

PALABRAS CLAVE: neuropublicidad - televisión - atención - emoción - género.

\section{ABSTRACT}

This paper shows the results of an experiment in neuroscience on a group of 20 people of both sexes, whose electrodermal activity was measured simultaneously while they

\footnotetext{
${ }^{1}$ Alejandro Tapia-Frade: Doctor en Publicidad por la Universidad Complutense de Madrid (2005). Acreditado por ANECA a Profesor Contratado Doctor, tiene un sexenio de investigación por ACSUCYL. Profesor adjunto en la Universidad Loyola Andalucía.
} 
Tapia- Frade, A. y del Toro-Acosta, A. Semidesnudo, género y otros factores en publicidad televisiva. Un acercamiento desde la neurociencia

were watching TV spots. Also, we paid special attention to the analysis of gender differences and their attentional and emotional activation patterns. The results show that spots containing half-naked models or models only in underwear are significantly higher in average attendance (in both sexes) with respect to those without this feature. In addition, we also concluded that there were significant differences in relation to the familiarity of the audience with the brand in both sexes too (more attention is paid to known brands) Finally, it was also possible to emphasize that attentional activation occurs to a greater extent at the beginning and end of the spot, although it is more clearly seen in the case of women. Likewise, a common pattern of attentional activation may be suggested when a sound rupture occurs.

KEY WORDS: neuroadvertising - television - attention - emotion - gender.

\section{SEMIDESNUDO, GÊNERO E OUTROS FATORES NA PUBLICIDADE TELEVISIVA. UMA APROXIMAÇÃO DESDE A NEUROCIÊNCIA}

\section{RESUME}

Este trabalho mostra os resultados de um experimento de neurociência realizado sobre um grupo de 20 pessoas de ambos sexos, nas quais foram medidas suas atividades eletro dérmicas de forma simultânea enquanto viam spots de televisão. Prestando atenção especialmente na analises de diferenças de gênero e seus padrões de ativação de atenção e emoção. Os resultados mostram que os spots que mostravam modelos semidesnudos ou com roupa intima resultavam significativamente superior na atenção em ambos sexos. Também foi concluído diferenças significativas em relação a familiaridade dos telespectadores com a marca em ambos sexos (as marcas conhecidas chamam mais a atenção). Finalmente, destacou-se também que a ativação da atenção se produz em maior medida ao início e finalização do spot, apesar que também se aprecia, mas claramente no caso entre as mulheres. De modo igual, pode-se sugerir um padrão comum de ativação da atenção quando se produz a ruptura relevante do registro sonoro.

PALAVRAS CHAVE: neuropublicidade - televisão - atenção - emocional - gênero.

\section{Como citar el artículo:}

Tapia- Frade, A. y del Toro-Acosta, A. (2019). Semidesnudo, género y otros factores en publicidad televisiva. Un acercamiento desde la neurociencia. [Half-naked, gender and other factors on television spots. A neuroscience approach]. Vivat Academia. Revista de Comunicación, 147, 1-21. http://doi.org/10.15178/va.2019.147.1-21. Recuperado de http://www.vivatacademia.net/index.php/vivat/article/view/1101

Vivat Academia. Revista de Comunicación. 15 junio 2019 /15 septiembre 2019, nº 147, 1-21 
Tapia- Frade, A. y del Toro-Acosta, A. Semidesnudo, género y otros factores en publicidad televisiva. Un acercamiento desde la neurociencia

\section{INTRODUCCIÓN}

El recurso a la sensualidad, a la belleza sugerida, es sin duda uno de los más utilizados en publicidad. Tanto es así que se ha llegado a hablar del concepto de marketing sensual (Moreno, 2004).

En ese contexto es relevante señalar la abundancia la literatura acerca de la publicidad y la percepción de los estereotipos de género manejados por ella. Así, Moreno y Martínez (2012) señalan que la producción estereotípica de la publicidad en televisión otorga a los hombres valores como fuerza, arrogancia, valentía, riesgo, temeridad o dominio, mientras que para la mujer reserva significados asociados a la belleza, la dulzura, la ternura, el cariño o la reflexión.

Anteriormente, Soloaga (2007) en un estudio sobre publicidad de marcas de lujo en España señaló que la publicidad ensalza valores asociado a una mujer entendida como objeto de deseo que persigue belleza y juventud como valores esenciales.

Sin ánimo de extenderse en la cuestión, son sin duda imprescindibles las referencias a los estudios de David y Johnson (1994), Kang (1997), David, Morrison, Johnson y Ross (2002), Lindner (2004) y Dittman y Howard (2004), en una línea teórica similar a lo antes descrito.

No obstante, representaciones publicitarias más vanguardistas tienden a una representación más igualitaria de la mujer, en la que la mujer asume roles tradicionalmente vedados a su condición, asociándola con el poder, la capacidad, la fortaleza, la realización personal e incluso la agresividad. (Garrido Lora, 2007, p. 67).

También hay que mencionar en opinión del mismo autor que la representación de la masculinidad ha sufrido una importante transformación en los mensajes publicitarios, que abandona el sólido perfil antes mencionado en beneficio de una imagen más plural, en la que encuentra cabida la sensibilidad, la preocupación estética o la ocupación en tareas domésticas o propias de la paternidad, otrora patrimonio estereotípico de la mujer. (Garrido Lora, 2007, p. 67).

No obstante, cabe también preguntarse si esos roles estereotípicos promovidos por la publicidad despiertan en efecto la atención de los espectadores, los destinatarios al fin de tal publicidad.

La investigación psicobiológica de los destinatarios podría ser un camino válido a tal estudio. De hecho, se ha llegado a mencionar que si se analizara a una persona que visualiza un anuncio publicitario, podrían controlarse tres variables fundamentales del

Vivat Academia. Revista de Comunicación. 15 junio 2019 /15 septiembre 2019, nº 147, 1-21 
proceso, que son la atención, la emoción y la memoria, con lo que se podría optimizar el mismo para que causara mayor impacto. (Torreblanca et al., 2012).

Además, la literatura en relación al estudio de los efectos de la publicidad mediante técnicas neurocientíficas es cada vez más abundante.

Así, en un trabajo sobre familiaridad en marcas, se realizaron dos experimentos para analizar la activación neurofisiológica, la latencia de respuesta a tal activación, y la elección de marcas nuevas y marcas familiares. Los resultados muestran que (1) la elección de nuevas marcas (en comparación con la elección de marcas conocidas) es precedida por un aumento de la activación de la corteza prefrontal, medido mediante resonancia magnética funcional (fMRI); (2) la elección de marcas nuevas se asocia con la latencia de respuesta mayor que las marcas conocidas; y (3) el estado de ánimo positivo mejora la latencia de respuesta de la elección de nuevas marcas en comparación con las marcas conocidas. (Reimann, 2012).

Otro estudio, en este caso realizado sobre spots mediante electroencefalografía y respuesta galvánica a 28 personas puso de manifiesto diferencias significativas de atención y emoción entre géneros según las categorías de productos visualizadas (Vecchiato et al., 2014b). Otro, basada en la marca Coca Cola puso de relevancia la importancia de la música como elemento persuasor en el marketing audiovisual (Sanchez-Porras, 2013).

Otra investigación realizada sobre publicidad exterior puso de relevancia la creatividad y la atención como conductores fundamentales de éxito en publicidad, en concreto en relación a la mejora de recuerdo de marca. (Wilson, 2015, p. 232).

Finalmente, otro trabajo sobre spots en tv que usaba indicadores biométricos y eyetracker demostró el valor de la televisión como herramienta para atraer y mantener respuesta emocional. Además, dicha investigación puso de manifiesto que dicho engagement emocional se veía incrementado cuando los participantes del estudio combinaban visualización de contenidos similares en $t v$ y en internet de forma simultánea (Steele et al., 2013, p. 417).

En ese contexto, este artículo muestra los resultados de un experimento de neuropublicidad en el que se analizó simultáneamente la actividad electrodérmica de 20 personas de ambos sexos mientras visionaban spots en los que aparecían modelos semidesnudos o en ropa interior mezclados con otros que no poseían esta cualidad.

Además, en este análisis se testaron otros factores que pudieran ser relevantes en los procesos de activación atencional y emocional de dichos sujetos. En concreto, se testaron

Vivat Academia. Revista de Comunicación. 15 junio 2019 /15 septiembre 2019, nº 147, 1-21 
Tapia- Frade, A. y del Toro-Acosta, A. Semidesnudo, género y otros factores en publicidad televisiva. Un acercamiento desde la neurociencia

diferentes formatos comerciales, y se exploró qué papel juegan el conocimiento de la marca y el lenguaje de locución del spot en la activación de dichos procesos.

\section{OBJETIVOS}

Los objetivos de conocimiento propuestos para este trabajo son:

1. Medición simultánea de la atención (EDL) y emoción (EDR) de una muestra de población durante la emisión de un conjunto de spots mediante el examen de su actividad electrodérmica.

2. Establecimiento de diferencias significativas en razón de género en los patrones de activación de las señales de atención (EDL) y emoción (EDR) mediante el examen de su actividad electrodérmica respecto de los siguientes factores:
a) la tipología del spot
b) la aparición (o no) de modelos semidesnudos o en ropa interior
c) el idioma de locución del spot
d) la familiaridad con la marca y el spot.

\section{METODOLOGÍA}

La muestra, escogida mediante el método de muestreo por conveniencia, consta de 20 personas de entre 20 y 30 años de edad de ambos sexos (en proporción similar). Por tanto, es una muestra cuyos resultados deben considerarse exploratorios.

Los spots emitidos y posteriormente analizados se muestran en tabla siguiente.

Tabla 1. Spots emitidos.

\begin{tabular}{|l|l|l|l|l|l|}
\hline Spot & Situación & Tipología & $\begin{array}{l}\text { Semidesnudo/ } \\
\text { Ropa Interior }\end{array}$ & $\begin{array}{l}\text { Emitido en } \\
\text { España }\end{array}$ & Idioma \\
\hline AMC Policy Minioms & $00: 01-00: 48$ & Cómico & NO & NO & ENG \\
\hline GreenPeace vs Lego & $00: 49-02: 33$ & Musical & NO & NO & ENG \\
\hline El Corte Inglés & $02: 34-03: 27$ & Musical & SI & SI & ES \\
\hline Target.com & $03: 28-03: 57$ & Cómico & SI & NO & ENG \\
\hline Aurgi & $03: 58-04: 18$ & Jingle & SI & SI & ES \\
\hline Batman & $04: 19-04: 49$ & Cinematográfico & NO & NO & OTR \\
\hline Andes & $04: 50-05: 49$ & Jingle & NO & NO & ES \\
\hline $\begin{array}{l}\text { Dolce Gabanna The } \\
\text { One }\end{array}$ & $05: 50-06: 34$ & $\begin{array}{l}\text { Paisajista / } \\
\text { Preciosista }\end{array}$ & SI & SI & ENG \\
\hline Aquarius & $06: 35-07: 36$ & Cómico & NO & SI & ES \\
\hline Guitar Hero & $07: 37-08: 14$ & $\begin{array}{l}\text { Paisajista / } \\
\text { Preciosista }\end{array}$ & SI & NO & ENG \\
\hline Limón y Nada & $08: 15-08: 25$ & Cómico & NO & SI & ES \\
\hline Coca Cola & $08: 26-09: 26$ & Jingle & NO & SI & ENG \\
\hline
\end{tabular}

Vivat Academia. Revista de Comunicación. 15 junio 2019 /15 septiembre 2019, n 147, 1-21 
Tapia- Frade, A. y del Toro-Acosta, A. Semidesnudo, género y otros factores en publicidad televisiva. Un acercamiento desde la neurociencia

\begin{tabular}{|l|l|l|l|l|l|} 
Mango by Opticalia & Pa:29 $-09: 49$ & $\begin{array}{l}\text { Paisajista / } \\
\text { Preciosista }\end{array}$ & SI & NO & OTR \\
\hline Orange & $09: 50-10: 32$ & Cómico & NO & NO & ENG \\
\hline Victoria's Secret & $10: 33-11: 03$ & $\begin{array}{l}\text { Paisajista / } \\
\text { Preciosista }\end{array}$ & SI & NO & ENG \\
\hline Mercedes Benz & $11: 04-11: 24$ & Cómico & NO & SI & ES \\
\hline Freixenet & $11: 25-11: 55$ & Musical & NO & SI & ES \\
\hline Nike & $11: 56-13: 13$ & Motivacional & NO & NO & ENG \\
\hline Cabreiroa & $13: 14-13: 24$ & Cómico & NO & SI & ES \\
\hline Mango Rebel & $13: 25-13: 45$ & $\begin{array}{l}\text { Paisajista / } \\
\text { Preciosista }\end{array}$ & SI & SI & ENG \\
\hline Line & $13: 46-14: 06$ & Cómico & NO & SI & ES \\
\hline Heineken & $14: 07-14: 37$ & Cómico & NO & NO & ENG \\
\hline Nokia & $14: 38-15: 38$ & Cómico & NO & NO & ENG \\
\hline Sorteo El Niño & $15: 39-16: 04$ & Cómico & NO & SI & ES \\
\hline
\end{tabular}

Fuente: elaboración propia.

Así, se diferenció si en el spot aparecían modelos semidesnudos o en ropa interior.

Por otra parte, se clasificaron los spots de acuerdo a una tipología diseñada ad hoc. En concreto las posibilidades que se listaron para ambos tipos fueron los caracteres jingle, musical, cómico, paisajista/preciosista, Spices of life/costumbrista, motivacional y cinematográfico.

También se destacó la marca responsable del spot y si fue emitido en España, que puede relacionarse con la familiaridad a priori desarrollada con la marca y el spot.

Finalmente, también se señala el idioma de la locución del spot (español, inglés y otro o sin locución), ya que podría tener incidencia sobre la comprensión del spot, y ésta sobre la atención desplegada para entenderlo y la intensidad emocional desarrollada.

Se realizó un pase de 20 personas el día 22 de abril de 2015 en el salón de grados de la Universidad Europea Miguel de Cervantes, en Valladolid. A la entrada del recinto de colocaba un brazalete a los participantes, que enviaba la información de cada persona a una unidad central de proceso, encargada de los cálculos necesarios y su almacén.

El instrumento de medición analiza la actividad electrodérmica en tres tipos de señales:

1) La actividad tónica, vinculada a la atención (EDL, o electrodermal level). La unidad de medida usada es el sumatorio de la resistencia electrodérmica en Kilomnios $(\mathrm{K} \Omega)$. Por ellos, es fundamental para la interpretación señalar que a menor resistencia, mayor nivel de atención.

Vivat Academia. Revista de Comunicación. 15 junio 2019 /15 septiembre 2019, nº 147, 1-21 
Tapia- Frade, A. y del Toro-Acosta, A. Semidesnudo, género y otros factores en publicidad televisiva. Un acercamiento desde la neurociencia

2) La actividad fásica, vinculada a la emoción (EDR, o electrodermal response). En este caso, la unidad de medida es la media aritmética de la resistencia electrodérmica en Kilomnios $(K \Omega)$ de todos los participantes. Por ello, a mayor media, mayor intensidad de emoción. Es también destacable que el instrumento de medición detecta simplemente la presencia de emoción, pero no el tipo (positivo o negativo) de ésta (como pudiera hacer un casco de electroencefalografía, por ejemplo).

3) Una señal espontánea, no específica, aleatoria e independiente de cada individuo (NSA, o non specific activity). Esta señal por ser inespecífica y subjetiva se considera ruido, por lo que se compensó mediante la media aritmética global para posteriormente poder discriminarla y despreciarla.

Dicha tecnología, de nombre Sociograph, patente $n^{\circ}$ 9902767, se ha usado con resultados favorables en casos anteriores como Martínez Herrador, J. L. et al. (2008, 2012) y Aiger, M. et al. (2013), entre otros.

\section{RESULTADOS}

Previo al análisis, se debe señalar que la prueba se realizó atendiendo a un modelo de series temporales con fuerte autocorrelación, lo que supone la significativa incidencia de los valores anteriores de atención (EDL) sobre los posteriores. La emoción, por su carácter súbito, no presenta esta dependencia.

\subsection{Atención}

El nivel medio de atención para el conjunto de la muestra es de 176,0634 K $\Omega(244,7952$ en el caso de los hombres y 152,5265 en el caso de las mujeres), con una desviación típica de 20,4036 (siendo de 19,5325 en el caso de los hombres y 20,04831 en el caso de las mujeres).

Los coeficientes de curtosis $(-1,108)$ y asimetría $(0,110)$ de Fisher para el conjunto de la muestra sugieren una distribución de tipo platicúrtico con ligera asimetría a la derecha o positiva. En el caso de los hombres también cabe hablar de una distribución platicúrtica (Coef. Curtosis: $-0,822)$, menos acusada que en el caso de las mujeres $(-1,122)$, y más asimétrica hacia la derecha (Coef Asimetría Hombres: 0,337) que estas últimas $(0,085)$, que presenta una cuasi simetría.

Se debe también señalar antes de presentar en detalle los resultados que el nivel de atención se mide en función de la resistencia, de modo a que mayor valor en resistencia, menor nivel de atención, y viceversa. Con el objetivo de simplificar la lectura del gráfico posterior, se han invertido los valores, de modo que cuanto más próximo a cero el valor, mayor nivel de atención.

Vivat Academia. Revista de Comunicación. 15 junio 2019 /15 septiembre 2019, nº 147, 1-21 
Tapia- Frade, A. y del Toro-Acosta, A. Semidesnudo, género y otros factores en publicidad televisiva. Un acercamiento desde la neurociencia

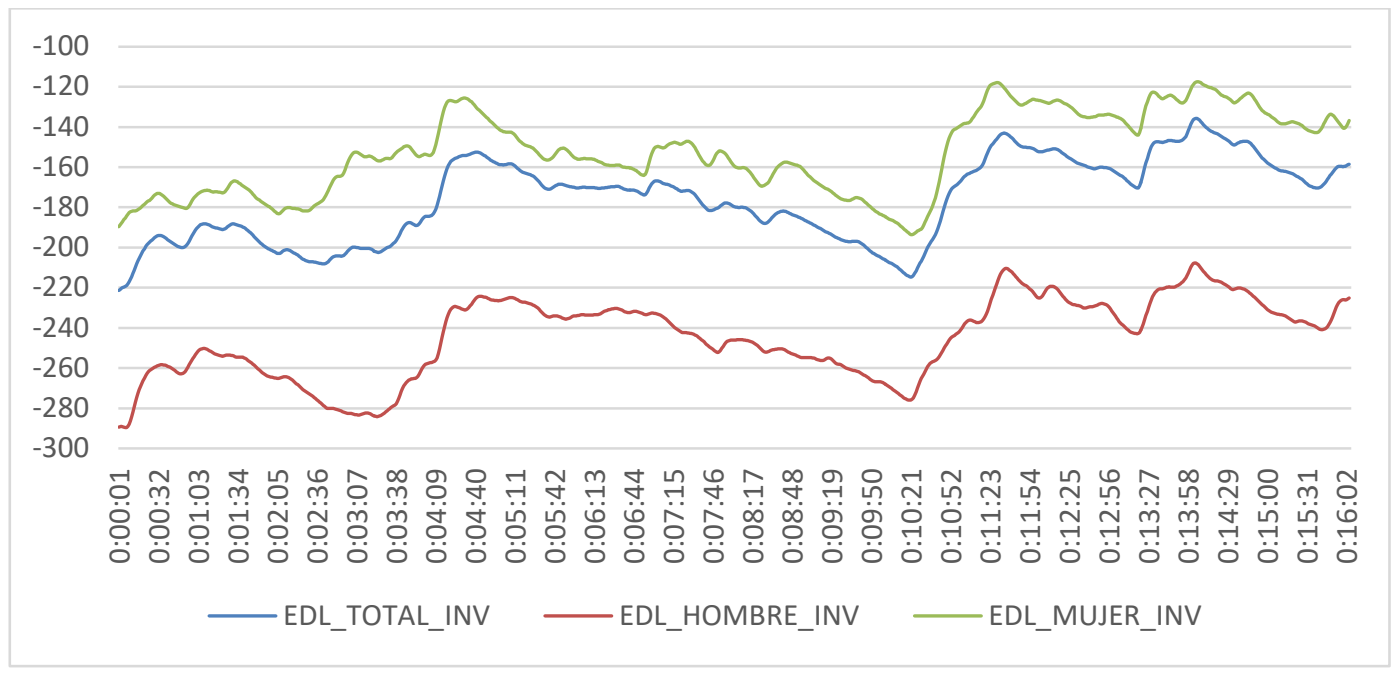

Gráfico 1. EDL (electrodermal level) de la emisión según sexo. $\left(-\sum K \Omega\right)$ Fuente: elaboración propia.

Así, cabe señalar una estructura global creciente con alteraciones visibles, que serán objeto de análisis posterior.

Si bien hay que hacer notar que, al usar la EDL un modelo de series temporales con fuerte autocorrelación, el análisis de la curva de atención (EDL) se debe basar en la estimación de las tasas de cambio de dicha variable, que pueden apreciarse en el gráfico 2. En este gráfico, los incrementos de nivel de EDL implican un aumento de la atención, y su decrecimiento una disminución. Por tanto, la característica más notoria de la serie es el crecimiento o decrecimiento de la curva y su intensidad en los distintos momentos de la emisión.

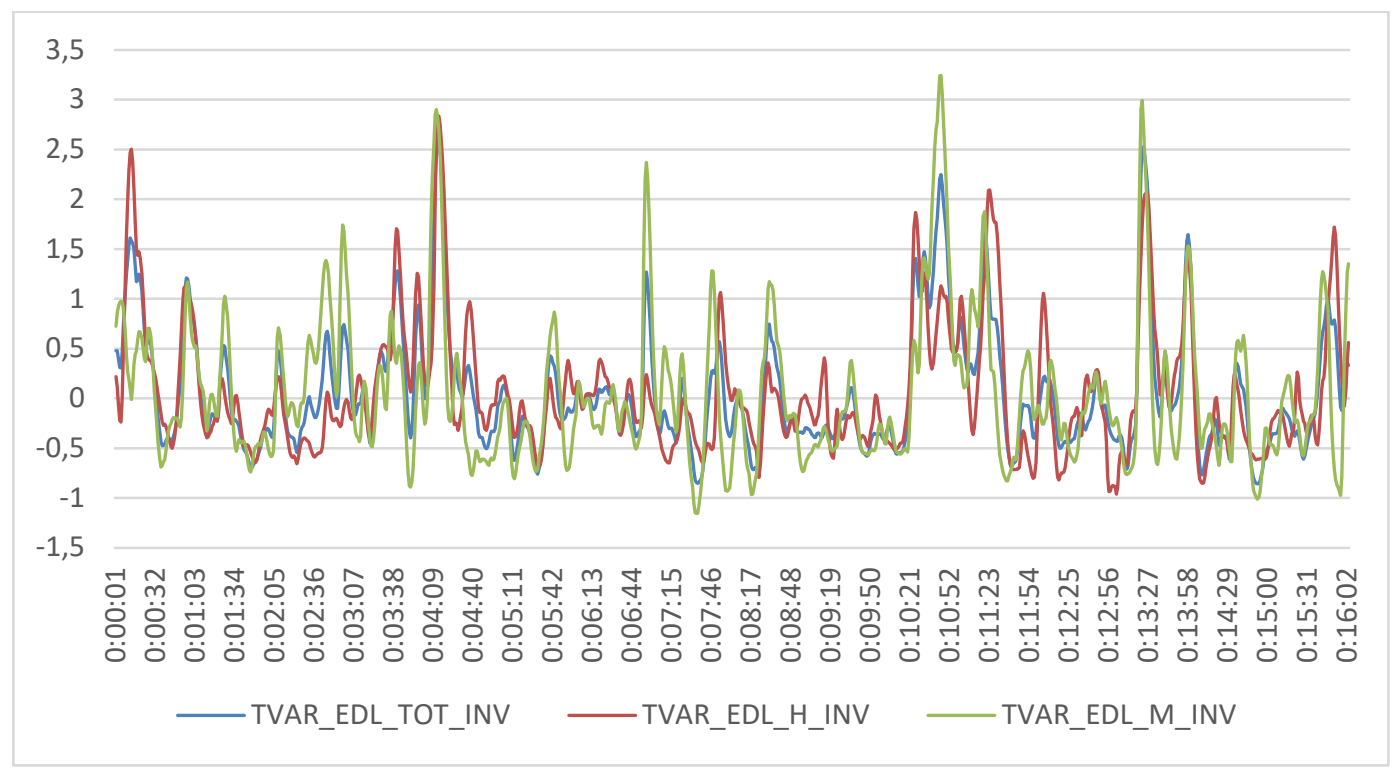

Gráfico 2. Tasa de Variación absoluta sobre EDL invertida de la emisión por sexo. (-K $\Omega$ ). Fuente: elaboración propia.

Vivat Academia. Revista de Comunicación. 15 junio 2019 /15 septiembre 2019, nº 147, 1-21 
Tapia- Frade, A. y del Toro-Acosta, A. Semidesnudo, género y otros factores en publicidad televisiva. Un acercamiento desde la neurociencia

Sin duda, pueden apreciarse puntos en los que dicha tasa de cambio sufre alteraciones reseñables, siendo éstos los momentos en que la atención se ve incrementada por un estímulo determinado. Los momentos de mayor alteración son los siguientes:

1) AMC Sprint. Mujeres (00:01-00:09) - Tasa Variación EDL $(\Delta K \Omega):$ 6,986 -. Es el Inicio del spot. En visual, se aprecian unos minioms sentados en el cine, y aparece un miniom morado, que se come una silla. En sonoro, una voz en off en inglés señala "this is Dave, he really enjoys going to the movies, and he is always considered with..." El miniom morado grita.

2) AMC Sprint. Hombres (00:09-00:25) - Tasa Variación EDL $(\Delta K \Omega): 27,785$-. En visual, el miniom morado se come un brazo de un sillón. Dave saca el móvil para apagarlo, pero el miniom morado se lo come. Posteriormente, el teléfono suena, Dave aprieta la barriga del miniom morado y el móvil se apaga.

3) Greenpeace Hombres y mujeres (00:53:01:04) - Tasa Variación EDL $(\Delta \mathrm{K} \Omega)$ : H $(11,126)$ M $(7,765)$-. Momentos iniciales del spot. En visual, se aprecian planos largos de figuras de Lego (dos osos, unos lobos, un esquimal pescando y un grupo de jugadores de hockey hielo, una gaviota. En sonoro, una canción instrumental comienza. No hay voz.

4) El Corte Inglés. Mujeres (02:40 - 02:51) -Tasa Variación EDL $(\Delta K \Omega)$ : 12,227. Momentos iniciales del spot. En visual, una mujer sale de una habitación. Se aprecian planos largos (más abundantes) y medios de una mujer vestida de diversas maneras. En sonoro, suena un portazo, y el inicio de una canción.

5) Target.com. Hombres (03:38 - 03:48) - Tasa Variación EDL $(\Delta K \Omega): 12,357$-. En visual, Se ve a Darth Vader sentado en una silla. Suena una puerta y aparece Heidi Klum, que se sienta al lado. Dath Vader la mira. En sonoro, lo único destacable es que la respiración de Dath Vader se acelera con la presencia de Heidi Klum.

6) Aurgi. Hombres y mujeres (04:07 - 04:22) - Tasa Variación EDL $(\Delta K \Omega)$ : H $(26,690)$ M $(26,859)$ - . En visual, sobre fondo verde, se aprecia a Rebeca, vestida sensualmente, cantando. El tramo de incremento llega al final del spot. En sonoro, suena una canción muy estridente.

7) Aquarius. Mujeres (06:53 - 07:01) - Tasa Variación EDL $(\Delta K \Omega)$ : 14,093 -. En visual, se aprecian planos largos de una mujer, un bodegón de verduras, un señor mayor durmiendo sentado, una mujer mojada, un hombre jugando al ping pong y unos niños corriendo. En sonoro, una voz en off relata la vida de un pueblo.

Vivat Academia. Revista de Comunicación. 15 junio 2019 /15 septiembre 2019, nº 147, 1-21 
Tapia- Frade, A. y del Toro-Acosta, A. Semidesnudo, género y otros factores en publicidad televisiva. Un acercamiento desde la neurociencia

8) Orange. Hombres (10:23 - 10:32) - Tasa Variación EDL $(\Delta K \Omega): 13,689$-. Se trata de la conclusión del spot. En visual, la frase "life is full of choices. Have you made the right one?" se aprecia sobre fondo negro. Finaliza el spot con el logo de Orange. En sonoro, se advierte una canción instrumental. No hay voz.

9) Victoria Secret. Hombres y mujeres $(10,33$ - 11,03) - Tasa Variación EDL $(\Delta K \Omega): H$ $(20,811)$ M $(46,261)$-. Atención incrementada durante todo el spot. En visual, se aprecian hasta 8 mujeres en ropa interior alternando planos cortos y largos. En sonoro, se reproduce una canción sensual.

10) Mercedes Benz. Hombres y mujeres (11:08 - 11:24) - Tasa Variación EDL ( $\Delta K \Omega): H$ $(11,189)$ M $(17,781)$-. En visual, se aprecia a un chico y una chica sentados en un sofá hablando. En sonoro, su conversación es la protagonista. En el caso de las mujeres, son los momentos iniciales del spot. En el caso de los hombres, el incremento de atención se produce hacia la mitad del mismo.

11) Freixenet. Hombres (11:25 - 11:34) - Tasa Variación EDL $(\Delta K \Omega):$ 14,914 -. Es el inicio del spot. En visual, un grupo de mujeres vestidas de color dorado bailan al son de una canción. En sonoro, la canción, de Bisbal, tiene un ritmo intenso.

12) Cabreiroa. Hombres y mujeres (13:20 - 13:24) - Tasa Variación EDL $(\Delta K \Omega): H$ $(6,877) \mathrm{M}(12,016)$-. Es el cierre del spot. En visual, se aprecia la parte delantera de una bicicleta con una botella de la marca. En sonoro, una voz en off femenina dice "Cabreiroa, agua equilibrada para gente que no para."

13) Mango. Hombres y mujeres (13:25 - 13:34) - Tasa Variación EDL $(\Delta K \Omega)$ : H $(14,719) M$ $(8,908)$-.Inicio del spot. En visual, se aprecian planos cortos de la cara de un modelo en blanco y negro. Solo en el caso de los hombres, el modelo se quita la camisa. En sonoro, el spot se inicia con una canción muy estridente.

14) Line. Hombres y mujeres (13:54 - 14:03) - Tasa Variación EDL $(\Delta K \Omega)$ : H $(9,540) \mathrm{M}$ $(9,457)$-. En visual, se aprecia a un hombre con un teléfono y cara de pánico. Se alternan planos largos del hombre tirado en el suelo con planos cortos de él y otro hombre. En sonoro, se inicia una parte con una batería.

15) Sorteo "El Niño". Hombres (15:47 - 15:57) - Tasa Variación EDL ( $\Delta K \Omega): 13,414$-. En visual, se aprecia a una pareja de ancianos sentados en un sofá. En sonoro, el anciano hombre relata lo pesado que ha sido para ellos aguantar a toda la familia durante las navidades.

Analizados los tramos de incremento de EDL, podemos señalar que son más frecuentes en tramos iniciales y finales de los spots en el caso de las mujeres (6 de 9

Vivat Academia. Revista de Comunicación. 15 junio 2019 /15 septiembre 2019, nº 147, 1-21 
Tapia- Frade, A. y del Toro-Acosta, A. Semidesnudo, género y otros factores en publicidad televisiva. Un acercamiento desde la neurociencia

ocasiones), pero no en el de los hombres (5 de 11 casos). En términos sonoros, parece advertirse un patrón común de iniciación de los tramos con un cambio de registro sonoro relevante respecto de los momentos anteriores, que puede apreciarse con mayor claridad en el caso de las mujeres (se da esta circunstancia en 7 ocasiones frente a 2 que no se dan, aunque en una persiste una canción con un ritmo muy elevado) que en los hombres (se da en 8 ocasiones frente a 4 en que no se da, aunque en 2 éstas se producen en el contexto de una canción de ritmo muy elevado).

Por otra parte, cabe señalar que 5 de los 8 spots que presentan modelos semidesnudos o en ropa interior presentan alteraciones atencionales significativas, frente a 9 de los 16 que no poseen esta característica.

Al margen de los momentos en que la atención se ve súbitamente incrementada, cabe señalar qué spots presentan un balance general de EDL positivo (siendo por ello spots captadores de atención) y cuales un balance negativo (siendo por ello spots que distraen o dispersan la atención).

Los resultados pueden apreciarse en la tabla siguiente.

Tabla 2. Balance en Tasa variación EDL $(\Sigma K \Omega)$.

\begin{tabular}{|l|l|l|l|}
\hline & & & \\
Spot & Total & Hombres & Mujeres \\
\hline AMC SPRINT & 21,714 & 26,76975 & 10,178875 \\
\hline Andes.- El imbancable & $-13,776$ & $-10,3285$ & $-15,323$ \\
\hline Aquarius.- El pueblo & $-6,923875$ & $-14,368375$ & 4,50625 \\
\hline Aurgi & 25,704 & 25,610375 & 26,599125 \\
\hline Batman Arkham Knight & 5,72775 & 9,8875 & $-7,553875$ \\
\hline Cabreiroa & 8,225 & 6,209875 & 8,974875 \\
\hline Coca Cola.- Can't beat the feeling & $-8,159375$ & $-6,89675$ & $-6,307875$ \\
\hline Dolce Gabana.- The One & $-1,371125$ & 4,4415 & $-8,8235$ \\
\hline El Corte Inglés.- Primavera & 5,394375 & $-9,121875$ & 23,428125 \\
\hline Freixenet & $-1,232875$ & 4,543875 & $-7,70175$ \\
\hline GreenPeace.- Lego is Awesome & $-7,5215$ & $-11,51325$ & $-0,4865$ \\
\hline Guitar Hero.- Heidi Klum & $-4,0495$ & $-1,176875$ & $-6,92125$ \\
\hline Heineken.- Jenifer Aniston & $-11,8685$ & $-11,641$ & $-9,52175$ \\
\hline Limon \& Nada & $-6,78825$ & $-5,032125$ & $-7,63875$ \\
\hline Line & 10,6785 & 11,197375 & 6,908125 \\
\hline Mango.- Rebel hero & 13,4785 & 16,158625 & 7,65625 \\
\hline Mercedes Benz & 15,3615 & 12,368125 & 19,446875 \\
\hline Nike & $-18,07925$ & $-21,039375$ & $-14,440125$ \\
\hline Nokia Lumia & $-22,39475$ & $-19,201$ & $-15,906625$ \\
\hline Opticalia & $-5,67$ & $-7,1505$ & $-3,836$ \\
\hline Orange.- Wedding & $-1,32125$ & 3,660125 & $-7,9065$ \\
\hline Sorteo Niño 2014 & 11,930625 & 14,368375 & 6,057625 \\
\hline & & & \\
\hline
\end{tabular}

Vivat Academia. Revista de Comunicación. 15 junio 2019 /15 septiembre 2019, nº 147, 1-21 
Tapia- Frade, A. y del Toro-Acosta, A. Semidesnudo, género y otros factores en publicidad televisiva. Un acercamiento desde la neurociencia

\begin{tabular}{|l|l|l|l|} 
Target.com & 15,4 & 22,73425 & 2,261 \\
\hline Victoria Secret & 38,41425 & 23,818375 & 49,177625 \\
\hline
\end{tabular}

Fuente: elaboración propia.

Así, en el caso de las mujeres destaca sobremanera el desempeño del spot de Victoria Secret, y posteriormente los de Aurgi, El Corte Inglés y Mercedes Benz. Cabe destacar que 3 de los 4 tienen modelos desnudos o en ropa interior. En el lado opuesto, los spots que peor respuesta obtuvieron fueron los de Nokia Lumia, Cerveza Andes y Nike, no siendo ninguno de ellos de tipo sensual.

En el caso de los hombres, en el lado positivo cabe destacar las puntuaciones de AMC Sprint, Aurgi, Victoria Secret y Target.com -en este caso, 3 de los 4 tienen modelos semidesnudos-. De igual modo, los que peor respuesta obtuvieron fueron Nike, Aquarius y Andes -también es de destacar que al igual que en el caso de las mujeres, ninguno contiene dichos modelos-.

La tipología de los spots destacables tanto positivamente como negativamente es variada en ambos sexos, lo que no permite sugerir tendencia atencional hacia ninguna tipología en concreto.

En todo caso se realizaron pruebas de comparación (ANOVAS de un factor) para comprobar tal tendencia. Los resultados obtenidos señalan la inexistencia de una tipología estadísticamente superior al resto para los tres grupos estudiados (hombres, mujeres y todos los casos).

Al contrario, si se pudo contrastar (usando el mismo método señalado antes) que la tasa de cambio media en atención desarrollada por los spots en español fue significativamente superior a los emitidos en inglés o sin locución en el caso de las mujeres y en el grupo de todos los casos ( $\mathrm{p}=0,000$ y 0,001 respectivamente).

En el caso de los hombres $(p=0,009)$ no se obtuvieron diferencias significativas en los spot emitidos español e inglés, pero si ambos respecto de los spots sin locución.

Respecto de los spots emitidos y no emitidos en España, cabe destacar que pudieron contrastarse diferencias medias de EDL significativas (por medio de una prueba T) a favor de los spots emitidos en España en todos los casos estudiados ( $p=0,000,0,011$ y 0,000 para los todos los casos, hombres y mujeres respectivamente).

En el caso de los spots con actores semidesnudos o en ropa interior, las pruebas de comparación realizadas permiten señalar que la tasa de cambio media en atención (o lo que es lo mismo, los incrementos medios de atención prestada al spot) es

Vivat Academia. Revista de Comunicación. 15 junio 2019 /15 septiembre 2019, nº 147, 1-21 
Tapia- Frade, A. y del Toro-Acosta, A. Semidesnudo, género y otros factores en publicidad televisiva. Un acercamiento desde la neurociencia

significativamente superior en los spots con modelos semidesnudos o en ropa interior en todos los casos analizados (hombres, mujeres y todos, prueba $\mathrm{T}$ de diferencia de medias con $\mathrm{p}=0,000,0,006$ y 0,000 )

\subsection{Emoción}

El nivel medio de EDR presentado por la serie es de 0,1803163 K $\Omega$ en el caso de los hombres y 0,2040547 en el caso de las mujeres. Las desviaciones típicas son respectivamente 0,23505 para los hombres y 0,29650 para las mujeres, lo que sugiere mayor dispersión en el caso de las mujeres. Los coeficientes de apuntamiento para hombres $(17,465)$ y mujeres $(23,283)$ sugieren la presencia de forma leptocúrtica en ambas distribuciones (más acusada en el caso de las mujeres) y los coeficientes de asimetría (3,453 y 4,008 respectivamente) indican una distribución asimétrica a la derecha.

Por tanto, debemos concluir parecida intensidad en términos de emoción (EDR) a lo largo de la emisión en hombres y mujeres, lo cual no implica que los estímulos que inician el proceso de estimulación emocional sean los mismos, como más adelante veremos.

Por ello, el análisis del nivel de EDR en la serie temporal posterior pone de manifiesto cambios súbitos en la resistividad de la piel que se relacionan con reacciones a estímulos concretos, diferentes en hombres y mujeres, como puede apreciarse en el gráfico 3, que se muestra a continuación.

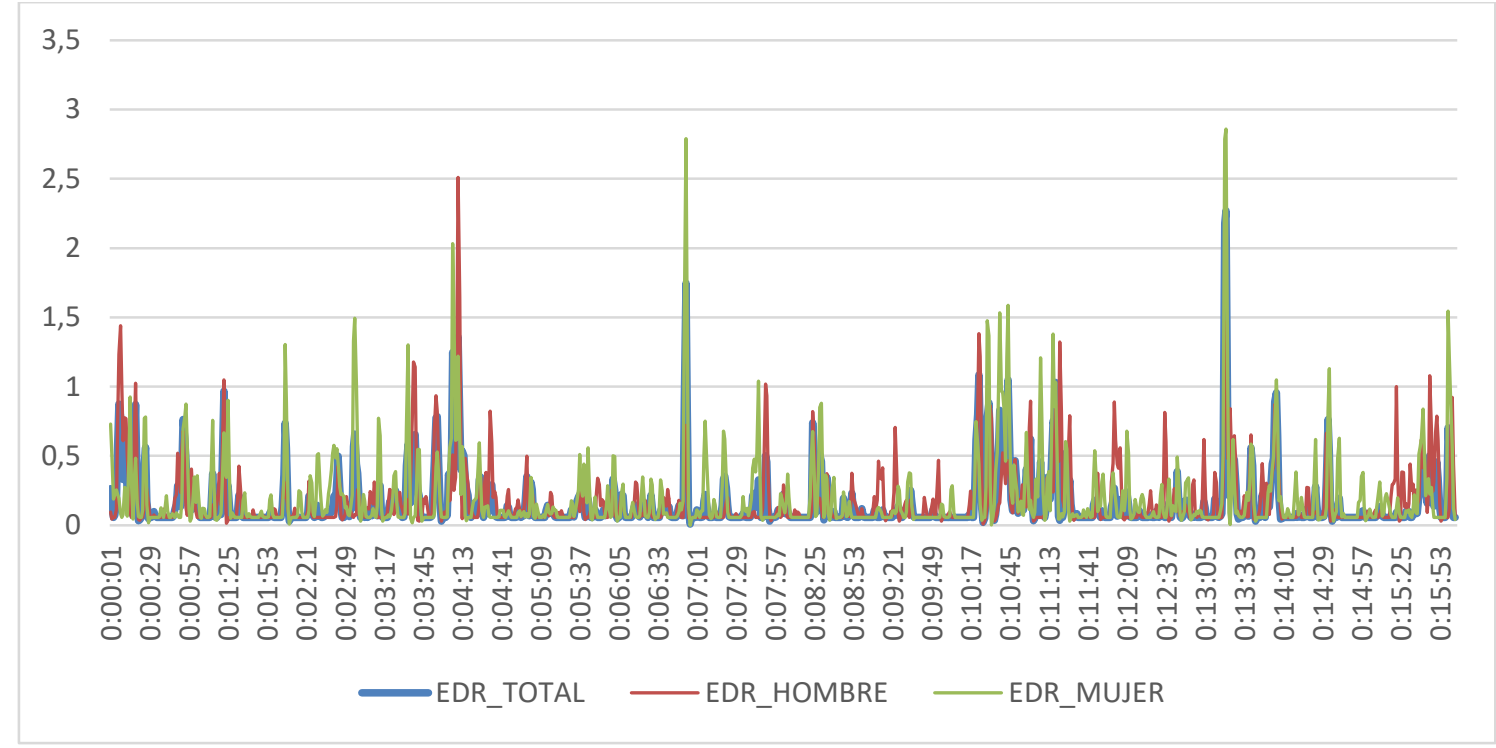

Gráfico 3. EDR (electrodermal response) de la emisión según sexo $(K \Omega)$. Fuente: elaboración propia.

Vivat Academia. Revista de Comunicación. 15 junio 2019 /15 septiembre 2019, nº 147, 1-21 
Tapia- Frade, A. y del Toro-Acosta, A. Semidesnudo, género y otros factores en publicidad televisiva. Un acercamiento desde la neurociencia

Así, pueden apreciarse cambios súbitos de registro en EDR para los hombres en los momentos 4: 09 a 4:11 (muy intenso), 00:06 a 00:09, 10:22 a 10:24 y 11:21 a 11:22 (de menor intensidad).

En el caso de las mujeres cabe destacar cambios muy relevantes en los momentos 4:06 a 4:07, 6:52 a 6:54 y 13:19 a 13:21. Además, aunque de menor importancia, también hay que señalar momentos de cambio en 2:55 a 2:57, 10:29 a 10:30, 10:37 a 10:44 y 15:59 a 16:01.

En la tabla posterior se describen los estímulos sonoros y visuales que inician estos cambios.

Tabla 3. Cambios en EDR (KR) y descripción del estímulo.

\begin{tabular}{|c|c|c|}
\hline Min / Seg. & $\operatorname{EDR}(\mathrm{K} \Omega)$ & Situación \\
\hline $\begin{array}{l}\text { 00:06 a 00:09 } \\
\text { a. } \mathrm{m} . \\
\end{array}$ & Hombres $(4,41)$ & $\begin{array}{l}\text { Spot de AMC Sprint. Un miniom morado grita y se come en brazo } \\
\text { de una butaca de cine. }\end{array}$ \\
\hline 02:55 a $02: 57$ & Mujeres $(3,843)$ & $\begin{array}{l}\text { Spot de El Corte Inglés. Plano largo de una modelo, la música } \\
\text { empieza un incremento de tono. }\end{array}$ \\
\hline 04:06 a 04:11 & $\begin{array}{l}\text { Mujeres }(3,581) \\
\text { Hombres }(5,428)\end{array}$ & Spot de Aurgi. Rebeca canta el jingle. La canción es de tipo Disco. \\
\hline 06:52 a 06:54 & Mujeres $(5,656)$ & $\begin{array}{l}\text { Spot de Aquarius. Una voz en off locuta la vida en un pueblo. En } \\
\text { visual se alterna un plano de un hombre jugando al ping pong, una } \\
\text { mujer mojada y una cigüeña que lleva un niño }\end{array}$ \\
\hline 10:22 a $10: 24$ & Hombres $(3,416)$ & $\begin{array}{l}\text { Spot de Orange. Finaliza el Spot. Fundido a negro con la frase "life } \\
\text { if full of choices" sobreimpresionada. }\end{array}$ \\
\hline 10:29 a $10: 30$ & Mujeres $(2,842)$ & $\begin{array}{l}\text { Spot de Orange. Final del spot. Sobre fondo negro, se aprecia la } \\
\text { marca sobreimpresionada. }\end{array}$ \\
\hline 10:37 a $10: 44$ & Mujeres $(8,066)$ & $\begin{array}{l}\text { Spot de Victoria Secret. Se alternan dos fundidos en negro con } \\
\text { frase "From Stockholm to Singapore" y "everyone knows" con } \\
\text { planos cortos y largos de } 3 \text { modelos en ropa interior. Suena una } \\
\text { canción sensual. }\end{array}$ \\
\hline 11:21 a $11: 22$ & Hombres $(2,141)$ & $\begin{array}{l}\text { Spot de Mercedes Benz. Final. En sonoro se aprecia un cambio de } \\
\text { registro relevante. En visual se ve un fundido a negro con la } \\
\text { palabra "algoestapasandoenmercedes.com". }\end{array}$ \\
\hline 13:19 a 13:21 & Mujeres $(6,707)$ & $\begin{array}{l}\text { Spot de Cabreiroa. Final del spot. Una voz en off dice "Cabreiroa. } \\
\text { Agua equilibrada para gente que no para". En visual se ve la parte } \\
\text { delantera de una bicicleta, una botella de Cabreiroa y al fondo el } \\
\text { mar. }\end{array}$ \\
\hline 15:59 a 16:01 & Mujeres $(6,671)$ & $\begin{array}{l}\text { Spot de Lotería del Sorteo del niño. Final del spot. Comienza a } \\
\text { sonar música y una voz en off masculina dice "Este año, premio } \\
\text { especial de } 40 \text { millones del sorteo del niño". En visual se aprecia a } \\
\text { dos ancianos sentados en un sofá. }\end{array}$ \\
\hline
\end{tabular}

Fuente: elaboración propia.

Vivat Academia. Revista de Comunicación. 15 junio 2019 /15 septiembre 2019, nº 147, 1-21 
Tapia- Frade, A. y del Toro-Acosta, A. Semidesnudo, género y otros factores en publicidad televisiva. Un acercamiento desde la neurociencia

En efecto, puede sugerirse un patrón común de cambio sonoro en los estímulos descritos en la tabla (se da en 6 de los 9 casos). También suelen darse en cambios visuales, tales como los momentos iniciales o finales de los spots.

Se pudo contrastar una relación objetiva entre tasas de cambio en EDL y picos en EDR para todos los casos estudiados (total, hombres y mujeres. En todos los casos el p= 0,000, y los Coef. Corr. de Pearson 0,380, 0,305 y 0,285 respectivamente). Es decir, se puede afirmar que cuando se produce un incremento emocional, también tiende a producirse un incremento atencional, y viceversa.

Además, 9 de los 10 picos emocionales se dan en tramos de incremento atencionales. Por ello, cabe señalar que existe un patrón de cambio conjunto entre atención y emoción. 4.

Otra cuestión es la emoción media generada por cada spot, que se muestra en la tabla

Tabla 4. Media EDR (K $\Omega)$ por spot y sexo.

\begin{tabular}{|c|c|c|c|}
\hline \multicolumn{2}{|l|}{ Spot } & EDR Hombre & EDR Mujer \\
\hline \multirow{2}{*}{ AMC SPRINT } & Media & 0,2624 & 0,1949 \\
\hline & Desv. típ. & 0,3500 & 0,2205 \\
\hline \multirow{2}{*}{ Andes .- El imbancable } & Media & 0,1116 & 0,1297 \\
\hline & Desv. típ. & 0,0842 & 0,1140 \\
\hline \multirow{2}{*}{ Aquarius.- El pueblo } & Media & 0,0986 & 0,2250 \\
\hline & Desv. típ. & 0,1065 & 0,4323 \\
\hline \multirow{2}{*}{ Aurgi } & Media & 0,4884 & 0,4912 \\
\hline & Desv. típ. & 0,6213 & 0,5492 \\
\hline \multirow{2}{*}{ Batman Arkham Knight } & Media & 0,1760 & 0,1244 \\
\hline & Desv. típ. & 0,1769 & 0,1146 \\
\hline \multirow{2}{*}{ Cabreiroa } & Media & 0,5107 & 0,7237 \\
\hline & Desv. típ. & 0,5156 & 1,0811 \\
\hline \multirow{2}{*}{ Coca Cola .- Can't Beat the feeling } & Media & 0,1437 & 0,1343 \\
\hline & Desv. típ. & 0,1318 & 0,1641 \\
\hline \multirow{2}{*}{ Dolce Gabana .- The One } & Media & 0,1214 & 0,1274 \\
\hline & Desv. típ. & 0,0806 & 0,1170 \\
\hline \multirow{2}{*}{ El Corte Inglés .- Primavera } & Media & 0,1208 & 0,2635 \\
\hline & Desv. típ. & 0,0842 & 0,3106 \\
\hline \multirow{2}{*}{ Freixenet } & Media & 0,1433 & 0,1595 \\
\hline & Desv. típ. & 0,1804 & 0,1616 \\
\hline \multirow{2}{*}{ GreenPeace .- Lego is Awesome } & Media & 0,1353 & 0,1846 \\
\hline & Desv. típ. & 0,1570 & 0,2326 \\
\hline \multirow{2}{*}{ Guitar Hero .- Heidi Klum } & Media & 0,1468 & 0,1670 \\
\hline & Desv. típ. & 0,2175 & 0,1993 \\
\hline \multirow{2}{*}{ Heineken .- Jenifer Aniston } & Media & 0,1301 & 0,1922 \\
\hline & Desv. típ. & 0,1558 & 0,2553 \\
\hline
\end{tabular}

Vivat Academia. Revista de Comunicación. 15 junio 2019 /15 septiembre 2019, nº 147, 1-21 
Tapia- Frade, A. y del Toro-Acosta, A. Semidesnudo, género y otros factores en publicidad televisiva. Un acercamiento desde la neurociencia

\begin{tabular}{|l|l|l|l|}
\multirow{2}{*}{ Limon \& Nada } & Media & 0,1787 & 0,1928 \\
\cline { 2 - 4 } & Desv. típ. & 0,2672 & 0,2203 \\
\hline \multirow{2}{*}{ Mine } & Media & 0,2522 & 0,2555 \\
\cline { 2 - 4 } & Desv. típ. & 0,2336 & 0,2797 \\
\hline \multirow{2}{*}{ Mercedes Benz } & Media & 0,1964 & 0,1825 \\
\cline { 2 - 4 } & Desv. típ. & 0,1883 & 0,1879 \\
\hline \multirow{2}{*}{ Nike } & Media & 0,3296 & 0,4180 \\
\cline { 2 - 4 } & Desv. típ. & 0,3347 & 0,4095 \\
\hline \multirow{2}{*}{ Nokia Lumia } & Media & 0,1666 & 0,1353 \\
\cline { 2 - 4 } & Desv. típ. & 0,1843 & 0,1233 \\
\hline \multirow{2}{*}{ Opticalia } & Media & 0,1337 & 0,1296 \\
\cline { 2 - 4 } Orange -- Dedding. típ. & 0,1530 & 0,1209 \\
\hline \multirow{2}{*}{ Sorteo Niño 2014 } & Media & 0,0935 & 0,1031 \\
\cline { 2 - 4 } & Desv. típ. & 0,0488 & 0,1019 \\
\hline \multirow{2}{*}{ Target.com } & Media & 0,2245 & 0,1898 \\
\cline { 2 - 4 } & Desv. típ. & 0,3190 & 0,3153 \\
\hline \multirow{2}{*}{ Victoria Secret } & Media & 0,3760 & 0,3532 \\
\cline { 2 - 4 } & Desv. típ. & 0,3072 & 0,3914 \\
\hline & Media & 0,3009 & 0,2158 \\
\cline { 2 - 4 } & Desv. típ. & 0,3373 & 0,2864 \\
\hline & Media & 0,2650 & 0,4591 \\
\cline { 2 - 4 } & Desv. típ. & 0,2023 & 0,4361 \\
\hline
\end{tabular}

Fuente: elaboración propia.

Así, en el caso de los hombres destaca el desempeño de Aurgi, Cabreiroa, Mercedes Benz y Lotería Nacional Sorteo El Niño. En el caso de las mujeres son destacables los spots de Aurgi, Cabreiroa, Mercedes Benz y Victoria Secret. En el polo opuesto, destacan negativamente para los hombres los spots de Opticalia, Aquarius, Andes, Dolce Gavana y El Corte Inglés. Destaca el hecho de que tres de los cinco casos son referidos a productos de moda. En el caso de las mujeres, destacan negativamente los spots de Opticalia, Andes, Batman Arkham Knight, Dolce Gavana y Nokia Lumia.

La tipología de los spots destacables, tanto positivamente como negativamente es variada en ambos sexos, lo que no permite sugerir tendencia emocional hacia ninguna tipología en concreto.

No obstante, se realizaron pruebas de comparación (ANOVAS de un factor) para corroborar tal tendencia. Los resultados obtenidos señalan la inexistencia de una tipología estadísticamente superior al resto para los tres grupos estudiados (hombres, mujeres y todos los casos).

Tampoco puede afirmarse nada en relación a la presencia de modelos semidesnudos o en ropa interior (de hecho, las pruebas de contraste realizadas no establecen diferencias significativas de cantidad media EDR respecto de esta cualidad de los spots).

Vivat Academia. Revista de Comunicación. 15 junio 2019 /15 septiembre 2019, nº 147, 1-21 
Al contrario, si se pudo contrastar (usando también ANOVAS de un factor) que la cantidad media de emoción desarrollada por los spots en español fue significativamente superior a los emitidos en inglés o sin locución en el caso de las mujeres y en el grupo de todos los casos $(p=0,000)$. Dicha diferencia no resultó significativa en el caso de los hombres $(p=0,060)$.

Finalmente, y al igual que en el caso anterior, pudieron contrastarse diferencias medias de EDR significativas (por medio de una prueba $\mathrm{T}$ ) a favor de los spots emitidos en España en los casos de mujeres y todos los casos ( $p=0,003$ y 0,030 respectivamente). Dicha diferencia, al igual que en caso anterior, no resultó significativa en el caso de los hombres $(p=0,338)$.

\section{DISCUSIÓN}

La activación atencional se producen en mayor medida al inicio y finalización del spot, aunque se aprecia más claramente en el caso de las mujeres. Asimismo, puede sugerir un patrón común de activación atencional cuando se produce una ruptura relevante de registro sonoro.

La tipología de los spots destacables en atención, tanto positivamente como negativamente es variada en ambos sexos, lo que no permite sugerir tendencia atencional hacia ninguna tipología en concreto. Es decir, no hay ninguna tipología que resulte significativamente superior al resto ni en hombres ni en mujeres.

No obstante, pudo relacionarse en todos los casos (hombres, mujeres y todos) mayores incrementos por término medio de atención prestada a los spots con modelos semidesnudos o en ropa interior.

Al igual, también se pudo contrastar que la tasa de cambio media en atención desarrollada por los spots en español fue significativamente superior a los emitidos en inglés o sin locución en el caso de las mujeres y en el grupo de todos los casos. En el caso de los hombres no se obtuvieron diferencias significativas en los spot emitidos español e inglés, pero si ambos respecto de los spots sin locución.

Respecto de los spots emitidos y no emitidos en España, cabe destacar que pudieron contrastarse diferencias medias de EDL significativas a favor de los spots emitidos en España en todos los casos estudiados. Por ello, cabe descartar que la familiaridad sea un factor en contra de la atención (al contrario de hecho).

En relación a los patrones de activación emocional, cabe destacar en primer lugar que se pudo contrastar una relación objetiva entre tasas de cambio en EDL y picos en EDR 
Tapia- Frade, A. y del Toro-Acosta, A. Semidesnudo, género y otros factores en publicidad televisiva. Un acercamiento desde la neurociencia

para todos los casos estudiados. Es decir, se puede afirmar que la activación de respuesta emocional se tiende a producir en un contexto de activación atencional.

De igual modo que con la EDL, no se hallaron diferencias significativas en ninguna tipología de spot respecto de las demás en ninguno de los colectivos estudiados (hombres y mujeres), pero si se pudieron contrastar diferencias significativas en cuanto al lenguaje del spot y si fue emitido en España, aunque sólo en el caso de las mujeres. Dicho de otro modo, la familiaridad con el spot y que esté locutado en español suponen para las mujeres dos factores a favor para la generación de contenido emocional.

La limitación más importante de esta investigación pasa por considerar el limitado tamaño de la muestra estudiada, de 20 personas. No obstante, estudios recientes señalan que la muestra elegida para un estudio de estas características es correcta, y los resultados fiables (Karmarkar, 2015; Orzan, 2015; Vecchiato, 2014; Martínez Herrador, 2012; Reimann, 2012).

Futuras líneas de investigación podrían ahondar en el contenido emocional generado por las diferentes tipologías de spots, o considerar el hecho del multitasking en los procesos y patrones de activación atencional y emocional mientras se ve la televisión y otros soportes -móviles, tablets, etc.

\section{REFERENCIAS}

Aiger, M.; Palacín, M., y Cornejo, J. M. (2013) La señal electrodérmica mediante Sociograph: metodología para medir la actividad grupal. Revista Internacional de Psicología Social: International Journal of Social Psichology, 28(3), 333-347.

David, P., y Johnson, M. (1998). The Role of Self in Third-Person Effects about Body Image. Journal of Communication, 48(4), 37-58.

David, P.; Morrison, G.; Johnson M., y Ross, F. (2002). Body Image, Race, and Fashion Models. Social Distance and Social Identification in Third-Person Effects. Communication Research. 29(3), 270-294.

Dittmar, H., y Howard, S. (2004). Professional hazards? The impact of models' bodysize on advertising effectiveness and women's body-focused anxiety in professions that do and do not emphasize the cultural ideal of thinness. British Journal of Psychology, $43,477-497$.

Garrido Lora, F. (2007). Estereotipos de género en Publicidad. La creatividad en la encrucijada sociológica. Creatividad y Sociedad, 11, 53-71.

Vivat Academia. Revista de Comunicación. 15 junio 2019 /15 septiembre 2019, nº 147, 1-21 
Tapia- Frade, A. y del Toro-Acosta, A. Semidesnudo, género y otros factores en publicidad televisiva. Un acercamiento desde la neurociencia

Karmarkar, U.; Yoon, C., y Plassmann, H. (2015). Marketers should pay attention to fMRI. Harvard Business Review. Recuperado de https://hbr.org/2015/11/marketersshould-pay-attention-to-fmri

Kang, M. (1997). The portrayal of women's images in magazine advertisements: Goffman's gender analysis revisited. Sex Roles. A Journal of Research. Gale Group.

Lindner, K. (2004). Images of women in general interest and fashion magazine advertisements from 1955 to 2002. Sex Roles. A Journal of Research, 51, pp. 409-421.

Martínez Herrador, J. L.; Garrido Martín, E.; Valdunquillo Carlón, M. I., y Macaya Sánchez, J. (2008). Análisis de la atención y la emoción en el discurso político a partir de un nuevo sistema de registro psicofisiológico y su aplicación a las ciencias políticas. DPSA. Documentos de trabajo del Departamento de Psicología Social y Antropología, 2. Recuperado de http://hdl.handle.net/10366/22533

Martínez Herrador, J. L.; Monge Benito, S., y Valdunquillo Carlón, M. I. (2012). Medición de las respuestas psicofisiológicas grupales para apoyar el análisis de discursos políticos. Tripodos, 29, 53-72.

Moreno, J. (2004). Iconos femeninos. Jordi González lanza el concepto de "marketing sensual", con el que sale en defensa de la publicidad ante los ataques que ésta recibe por determinados usos de la imagen de la mujer. Anuncios, 1079, 30.

Moreno, R. \& Martínez, M. M. (2012). Representación del hombre y de la mujer en la publicidad: análisis de los valores percibidos por el alumnado en función del género del protagonista del anuncio. Actas del I Congreso Internacional de Comunicación y Género. Sevilla, 5, 6 y 7 de Marzo de 2012. Sevilla: Facultad de Comunicación. Universidad de Sevilla.

Orzan, G.; Zara, I., y Purcarea, V. L. (2015). Neuromarketing techniques in pharmaceutical drugs advertising. A discussion and agenda for future research. Journal of medicine and life, 5(4), 428-432.

Reimann, M.; Castano, R.; Zaichkowsky, J., y Bechara, A. (2012) Novel versus familiar brands: an analysis of neurophysiology, response latency and choice. Marketing letters, 23(3), 745-759. doi: 10.1007/ s11002-012-9176-3

Sanchez-Porras, M. J. (2013). Music persuasión in audio-visual marketing. The example of Coca Cola. Historia y Comunicación Social, 18, 349-357. doi: 10.5209/rev_HICS.2013.v18.44333

Vivat Academia. Revista de Comunicación. 15 junio 2019 /15 septiembre 2019, nº 147, 1-21 
Tapia- Frade, A. y del Toro-Acosta, A. Semidesnudo, género y otros factores en publicidad televisiva. Un acercamiento desde la neurociencia

Soloaga, P. (2007). Valores y estereotipos femeninos en la publicidad de moda de lujo en España. Anàlisi, 35, 27-45.

Steele, A.; Jacobs, D.; Siefert, C.; Rule, R.; Levine, B., y Marci, C. (2013). Leveraging Synergy and Emotion In a Multi-Platform World A Neuroscience-Informed Model of Engagement. Journal of Advertising Research, 53(4), 417-430. doi: 10.2501/JAR-53-4-417430

Torreblanca, F.; Juarez, D.; Sempere, F., y Mengual, A. (2012). Neuromarketing: la emocionalidad y la creatividad orientadas al comportamiento del consumidor. 3Ciencias, revista de investigación. http:// hdl.handle.net/10251/34357.

Vecchiato, G. et al. (2014). How to measure cerebral correlates of emotions in Marketing relevant tasks. Cognitive Computation, 6(4), 856-871. doi: 10.1007/s12559-014-9304-x.

Wilson, R.; Baack, D., y Till, B. (2015). Creativity, attention and the memory for brands: an outdoor advertising field study. International Journal of Advertising, 34(2), 232-261. doi: 10.1080/02650487.2014.996117

\section{AUTORES}

\section{Alejandro Tapia-Frade}

Licenciado en Publicidad (1999) y Doctor en Marketing (2005), ambos por la Universidad Complutense de Madrid. Acreditado a las Figuras de Profesor Contratado Doctor y Profesor de Universidad Privada (ANECA y ACSUCYL), cuenta también con un tramo / sexenio de investigación (ACSUCYL), fruto de la publicación de aproximadamente cincuenta capítulos de libro y artículos en revistas indexadas. Docente universitario con ocho años de experiencia, antes en el departamento de Ciencias Sociales de la Universidad Europea Miguel de Cervantes, en Valladolid, y ahora en el Departamento de Comunicación y Educación de la Universidad Loyola Andalucía. Adicionalmente, realizado intercambios docentes en las universidades de Leiría y Guarda (Portugal), Konstanz (Alemania) y Arnhem (Holanda). Anteriormente a su etapa universitaria, durante siete años fue consultor de aplicaciones corporativas en entornos internet en varias empresas tecnológicas (Software AG, Everis).

ajtapia@uloyola.es

Orcid ID: http:/ / orcid.org/0000-0003-1071-4536

Google Scholar: https:// scholar.google.es/citations?user=CRPQeigAAAAJ\&hl=es

ResearchGate: $\underline{\text { https://www.researchgate.net/profile/Alejandro_Tapia_Frade }}$

\section{Andrés del Toro-Acosta}

Licenciado y Doctor en Publicidad (2015). Ha trabajado en las grandes agencias de Relaciones Públicas mundiales como Ogilvy en Madrid y Weber Shandwick en Madrid y

Vivat Academia. Revista de Comunicación. 15 junio 2019 /15 septiembre 2019, nº 147, 1-21 
Tapia- Frade, A. y del Toro-Acosta, A. Semidesnudo, género y otros factores en publicidad televisiva. Un acercamiento desde la neurociencia

Londres, ocupando diferentes puestos hasta la Dirección de Cuentas. Desde estas posiciones, he trabajado para clientes de diversos sectores como la Comisión y el Parlamento Europeo, Turespaña, Ford, Carrefour, Kellogg's, Coca-Cola, Instituto Danone, Ikea, Canon, Siemens, ANEABE o Cerámica de España, entre otros muchos. Profesor asociado e investigador especializado en estrategia en medios sociales, personal branding, gestión del contenido digital, comunicación corporativa, planificación estratégica y tendencias en comunicación en la Universidad Loyola Andalucía. Asimismo, ha realizado estancias de investigación en la Universidad de Rotterdam (Holanda).

atoro@uloyola.es

Vivat Academia. Revista de Comunicación. 15 junio 2019 /15 septiembre 2019, n 147, 1-21 